\title{
SUR LA TRANSMISSION EXPÉRIMENTALE DES TRYPANOSOMES DE PASSÉRIFORMES PAR CULICOÏDES
}

\author{
J. CHANDENIER, I. LANDAU, D. BACCAM
}

\begin{abstract}
RÉSUMÉ
Trente expériences de transmission de Trypanosoma chabaudi et de $T$. davidmolyneuxi par Culicoides nubeculosus ont été réalisées : elles ont apporté les renseignements suivants sur le cycle expérimental chez le vecteur et l'Oiseau : $a$ ) la spécificité pour l'hôte vertébré est faible; des transmissions croisées entre différentes espèces d'Oiseaux appartenant à deux familles (Estrildidae et Fringillidae) ont été réalisées; $b$ ) la parasitémie (1 à 10 parasites par $10 \mu$ l) et la morphologie du parasite chez les Oiseaux sont stables;

toute variation de la morphologie ou du taux de parasitémie entraînent l'impossibilité pour les Trypanosomes de se développer chez le vecteur; $c$ ) à l'exception de la voie trans-conjonctivale, toutes les autres voies d'infestation éprouvées (SC, IM, IP, PO) sont efficaces.

L'ensemble des observations amènent les auteurs à conclure que, dans des conditions normales, les Trypanosomes ne se multiplient pas chez l'Oiseau.
\end{abstract}

\section{SummaRy: Experimental transmission of passeriform Trypanosomes by Culicoides.}

Thirty transmission experiments of Trypanosoma chabaudi and T. davidmolyneuxi by Culicoides nubeculosus were made. The following informations, on the parasite's experimental cycle in the vector and the bird, were obtained: $a$ ) there was little specificity for the vertebrate host: transmission to different species of birds from two families (Fringillidae and Estrildidae) were achieved; b) rate of parasitaemia ( 1 to 10 parasites per $10 \mu \mathrm{l}$ ) and morphology of parasites in the blood were stable; trypanosomes became incapable of evolving in the vector when variations of the morphology or the rate of parasitaemia occurred; $c$ ) the transcunjunctival mode of infestation failed to produce an infection in the bird but all other ways (SC, IM, IP, PO) were successfully tested.

Following their observations, authors came to the conclusion that normally, Trypanosomes do not multiply in the bird.
Le développement de Trypanosomes aviaires chez Culicoïdes et leur transmission à de nouveaux Oiseaux ont été obtenus à deux reprises : T. avium de Turdus migratorius par Bennett en 1961 et $T$. johnbakeri de Psittacula roseata par Miltgen et Landau, en 1982.

Dans ce travail, nous exposons les conditions qui ont été nécessaires à la reproduction du cycle complet du parasite chez le vecteur et chez l'Oiseau.

\section{MATÉRIEL ET MÉTHODES}

\section{A - Culicoïdes}

Ce sont des Culicoides nubeculosus dont l'élevage a été entretenu au Muséum National d'Histoire Naturelle à Paris selon la technique de Boorman (1974) modifiée par Kremer.

Laboratoire de Protozoologie et Parasitologie comparée, EPHE et Laboratoire de Zoologie (Vers), associé au CNRS, Muséum National d'Histoire Naturelle, 61, rue Buffon, F 75231 Paris Cedex 05.

Accepté le : 25 février 1991.

\section{B - Trypanosomes}

Les expériences de transmission sont réalisées avec deux des cinq espèces rencontrées au cours de nos études (Chandenier et coll., 1988 a) sur les Trypanosomes de Passériformes : Trypanosoma chabaudi et $T$. davidmolyneuxi. Les formes métacycliques ( $\mathrm{mt}$ ) sont obtenues en sept jours chez le Culicoïde.

C - Oiseaux

Les Oiseaux sont des Passériformes, soit d'origine sauvage et capturés en Afrique sub-saharienne (Serinus mozambicus, Estrilda melpoda et E. astrild), soit d'élevage (S. canaria et Poephyla guttata). Tous ont été achetés dans des oiseleries parisiennes.

D - Méthodes

Les méthodes d'examen du sang des Oiseaux, de gorgement des vecteurs et d'infestation expérimentale sont celles décrites antérieurement (Chandenier et coll., $1988 a$ et $b$ ).

La quantification de la parasitémie est réalisée par comptage des parasites présents dans $10 \mu \mathrm{l}$ de sang prélevé à la pipette automatique réglable. La parasitémie est classée en : "inférieure à 10 parasites pour $10 \mu \mathrm{l}$ de sang » ou «comprise entre 15 et 25 parasites pour $10 \mu \mathrm{l}$ de sang $"$.

Cinq voies d'inoculation sont éprouvées, seules ou en associa- 
tion : intra-péritonéale (IP), intra-musculaire (IM), souscutanée (SC), orale (PO) et trans-conjonctivale.

Des autopsies sont pratiquées et des fixations au Carnoy et des appositions de cerveau, foie, rate, rein, poumon, intestin, peau sont réalisées.

\section{RÉSULTATS}

\section{A - Infectivité POUR Les CulicoïDes}

\section{1 - Infectivité et parasitémie}

La parasitémie, au cours des infections naturelles ou expérimentales, est, en général, faible, inférieure à 10 parasites $/ 10 \mu \mathrm{l}$ de sang (fig. 1).

Lorsque le suivi est réalisé sur une assez longue période, on peut constater des modifications à partir de ce taux de base : les Trypanosomes disparaissent parfois totalement de la circulation pendant une période plus ou moins longue; ailleurs, au contraire, des pics de 15 à 25 parasites/10 $\mu \mathrm{l}$ sont observés (fig. 1).

Nous avons recherché, sans la trouver, une relation entre les fluctuations de la parasitémie et les saisons ou la température extérieure.

Nous avons également recherché une séquestration des parasites dans les capillaires des organes profonds. Les autopsies réalisées sur trois Oiseaux négatifs (l'un ayant toujours été négatif et les deux autres s'étant négativés) et celles pratiquées sur cinq Oiseaux positifs donnent les résultats suivants : 1) Oiseaux négatifs : aucun parasite n'est trouvé dans les organes; 2) Oiseaux positifs : les parasites sont trouvés essentiellement dans le poumon et le foie; ils sont peu nombreux et ont une morphologie identique à celle qu'ils présentent dans le sang périphérique. Il n'y a apparemment pas de concentration dans les capillaires profonds.

Quoi qu'il en soit, ces variations semblent intervenir sur l'infectivité des Trypanosomes pour les Culicoïdes.

En effet, tous les développements obtenus chez le vecteur correspondent à une parasitémie stable, évoluant entre 1 et 10 parasites $/ 10 \mu \mathrm{l}$ de sang.

Chaque fois qu'une élévation brusque de la parasitémie survient, les gorgements ne donnent aucun résultat. Ceci est observé, aussi bien à la suite de la réapparition d'une parasitémie qui avait disparu qu'après augmentation d'une parasitémie préexistante (fig. 1).

De même, les Trypanosomes ne se sont développés chez les Culicoïdes que lorsque la parasitémie était décelable lors de l'examen direct du sang à frais : six fois des insectes sont gorgés sans succès sur des Oiseaux devenus apparemment négatifs.

\section{2 - Infectivité et morphologie}

Dans la majorité des infections naturelles ou expérimentales que nous avons survies, la morphologie des Trypanosomes est très stable.
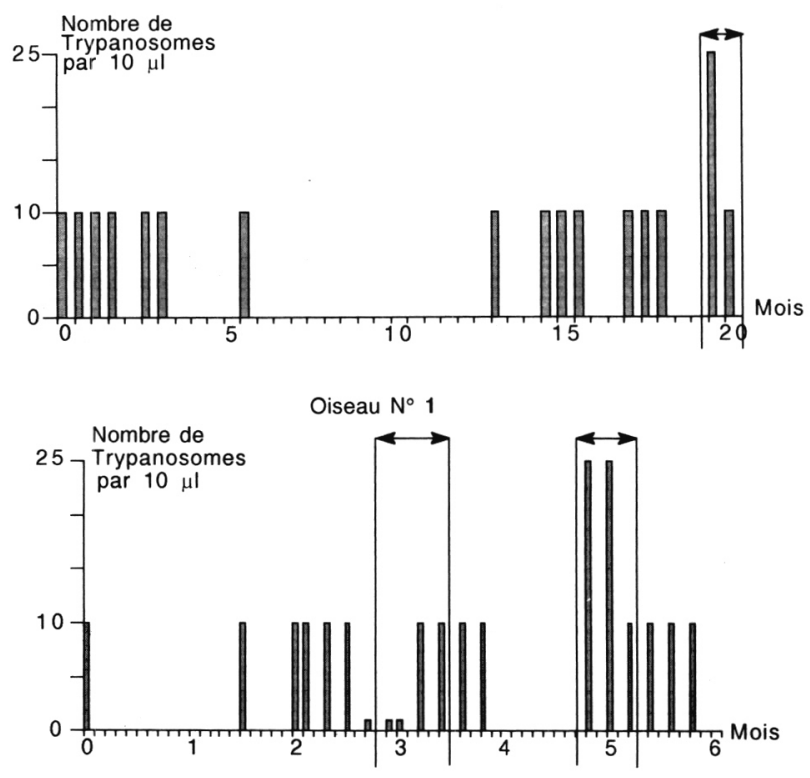

Oiseau $\mathrm{N}^{\circ} 2$

FIG. 1. - Infectivité des Trypanosomes pour les Culicoïdes en fonction de la parasitémie : l'infectivité des Trypanosomes pour les vecteurs disparaît lorsque la parasitémie subit une modification. Petits rectangles : aucun Trypanosome dans $10 \mu \mathrm{l}$ de sang; rectangles de taille moyenne : 1 à 10 Trypanosomes pour $10 \mu \mathrm{l}$; grands rectangles : 15 à 25 Trypanosomes pour $10 \mu \mathrm{l}$; double flèche horizontale : période pendant laquelle le Trypanosome perd son infectivité pour les Culicoïdes.

Pourtant, à la suite d'une transmission expérimentale, des différences morphologiques étaient apparues entre Trypanosoma chabaudi chez son hôte initial et celui observé dans la circulation d'un nouvel Oiseau (Chandenier et coll., $1988 \mathrm{~b}$ ). Bien que l'Oiseau receveur ait été capturé dans la nature, nous avions alors été convaincus de la réalité de la transmission expérimentale : vérifications répétées de la négativité de l'Oiseau receveur avant l'inoculation, apparition de la parasitémie dans les délais habituels, appartenance des deux Trypanosomes au même groupe de la classification de Baker (1976).

Au cours des 25 mois pendant lesquels l'Oiseau receveur a été suivi, sa parasitémie a évolué en quatre phases : parasitémie augmentant régulièrement pendant 3 mois, élevée pendant 18 mois (1 500 trypanosomes par $10 \mu \mathrm{l}$ ), décroissante pendant 2-3 mois, parasitémie stable et faible (1 à 10 trypanosomes par $10 \mu \mathrm{l}$ ) pendant 2 mois. Douze tentatives de transmission pendant la dernière période, au cours de laquelle le taux de parasitémie paraissait favorable à la transmission, restent sans succès.

3 - En résumé : les Trypanosomes sont infectants pour les Culicoïdes pendant les périodes où la parasitémie est stable et comprise entre 1 et 10 parasites dans $10 \mu \mathrm{l}$ de sang. Les modifications morphologiques de T. chabaudi, observées dans un cas, après transmission, représentent une anomalie qui sera discutée plus loin. 
TABlEAU I. - Tentatives de transmission de Trypanosoma davidmolyneuxi et de T. chabaudi par Culicoides nubeculosus : espèces d'Oiseaux, voies d'inoculation, quantité de formes métacycliqyues et délai d'apparition dans la circulation.

\begin{tabular}{|c|c|c|c|c|c|c|}
\hline $\begin{array}{l}N^{\circ} \text { trans }- \\
\text { mission }\end{array}$ & $\begin{array}{l}\text { Oiseau } \\
\text { donneur }\end{array}$ & $\begin{array}{l}\text { Oiseau } \\
\text { receveur }\end{array}$ & Voie & \begin{tabular}{|c|} 
Dose \\
métacycl. \\
\end{tabular} & Résultat & \begin{tabular}{c|} 
Délai \\
d'apparition \\
\end{tabular} \\
\hline T 1 & E. astrild & E. melpoda & I P & NC & - & - \\
\hline T 2 & E.astrild & E. melpoda & I P & $\mathrm{NC}$ & - & - \\
\hline T 3 & E.astrild & E. melpoda & IP & NC & - & - \\
\hline T 4 & E. astrild & E. melpoda & I P & NC & - & - \\
\hline T 5 & E. melpoda* & E. melpoda & SC & NC & - & - \\
\hline T 6 & E. melpoda* & E. melpoda & $\mathrm{PO}$ & $\mathrm{NC}$ & + & 3 jours \\
\hline T 7 & E. melpoda* & E.astrild & $\mathrm{IM}$ & NC & + & $?$ \\
\hline T 8 & E. melpoda* & S. canaria & $\mathrm{SC}+\mathrm{PO}$ & NC & - & - \\
\hline T 9 & E. melpoda* & P. guttata & $\mathrm{SC}+\mathrm{PO}$ & NC & - & - \\
\hline T 10 & S. mozambicus & S. canaria & toutes & NC & - & - \\
\hline $\mathrm{T} 11$ & S. mozambicus & S. canaria & toutes & NC & - & - \\
\hline T 12 & S. mozambicus & S. canaria & toutes & NC & + & 1 jour \\
\hline T 13 & S. mozambicus & S. canaria & toutes & 50000 & + & 6 heures \\
\hline T 14 & S. mozambicus & S. canaria & I P & 12500 & - & - \\
\hline T 15 & S. mozambicus & S. canaria & $\mathrm{PO}$ & 12500 & - & - \\
\hline T 16 & S. mozambicus & S. canaria & toutes & 50000 & + & 4 jours \\
\hline T 17 & S. mozambicus & P. guttata & toutes & 100000 & + & 2 jours \\
\hline T 18 & S. mozambicus & P. guttata & $\mathrm{PO}$ & 50000 & + & 2 jours \\
\hline T 19 & S. mozambicus & S. mozambicus & toutes & 40000 & - & \\
\hline T 20 & S. canaria & S. canaria & toutes & 36000 & + & 4 jours\# \\
\hline T 21 & P. guttata & P. guttata & toutes & 40000 & - & - \\
\hline T 22 & P. guttata & P. guttata & $œ$ il & 20000 & - & - \\
\hline T 23 & P. guttata & P. guttata & $\mathrm{PO}$ & 25000 & - & - \\
\hline T 24 & P. guttata & P. guttata & $œ i l$ & 25000 & - & - \\
\hline T 25 & P. guttata & P. guttata & $\mathrm{PO}$ & 25000 & - & - \\
\hline T 26 & P. guttata & P. guttata & $œ$ il & 50000 & - & - \\
\hline T 27 & P. guttata & P. guttata & I P & 30000 & + & 4 jours \\
\hline T 28 & P. guttata & P. guttata & I P & 25000 & - & - \\
\hline T 29 & P. guttata & P. guttata & SC & 25000 & + & 3 jours\# \\
\hline T 30 & P. guttata & P. guttata & toutes & 30000 & + & 3 jours \\
\hline
\end{tabular}

* Oiseau parasité par $T$. chabaudi. Voies : IP = intrapéritonéale, $\mathrm{SC}=$ sous-cutanée, $\mathrm{PO}=$ per os, $\mathrm{IM}=$ intra-musculaire, œil $=$ transconjonctivale.

- Total duquel a été soustrait la quantité utilisée par voie trans-conjonctivale, apparemment inefficace.

NC Non compté.

? Positif au premier contrôle, 20 jours après l'inoculation.

\# Négatif à 24 heures.

\section{B - Infectivité pour les Oiseaux}

Plusieurs voies d'inoculation ont été éprouvées et pour certaines expériences, une estimation du nombre de formes infectantes a pu être effectuée.

Toutes les expériences sont détaillées dans le tableau I où un numéro d'ordre de $\mathrm{T} 1$ à $\mathrm{T} 30$ est attribué à chaque tentative de transmission.

La figure 2 regroupe les transmissions réussies.

\section{1 - Période pré-patente}

Quelles que soient les modalités de l'infestation, la parasitémie est décelée chez l'Oiseau receveur entre 6 heures (T13) et 4 jours (T16, T20 et T27) après l'inoculation. Nous n'avons pas trouvé de relation entre le nombre de formes métacycliques injectées, la voie d'inoculation et la durée de la période pré-patente.

\section{2 - Spécificité}

Elle n'est pas très étroite. Des transmissions entre Oiseaux soit appartenant à la même espèce (T6, T20, T27, T29 et T30), soit à des espèces différentes d'une même famille (T7, T12, T13 et T16) ou de deux familles différentes (Fringillidae et Estrildidae, T17, T18) ont pu être réalisées.

\section{3 - Voies d'inoculation}

Toutes les voies éprouvées, seules ou en association, se sont révélées efficaces, la voie trans-conjonctivale exceptée.

Celle-ci est testée trois fois en trois mois avec $T$. david- 

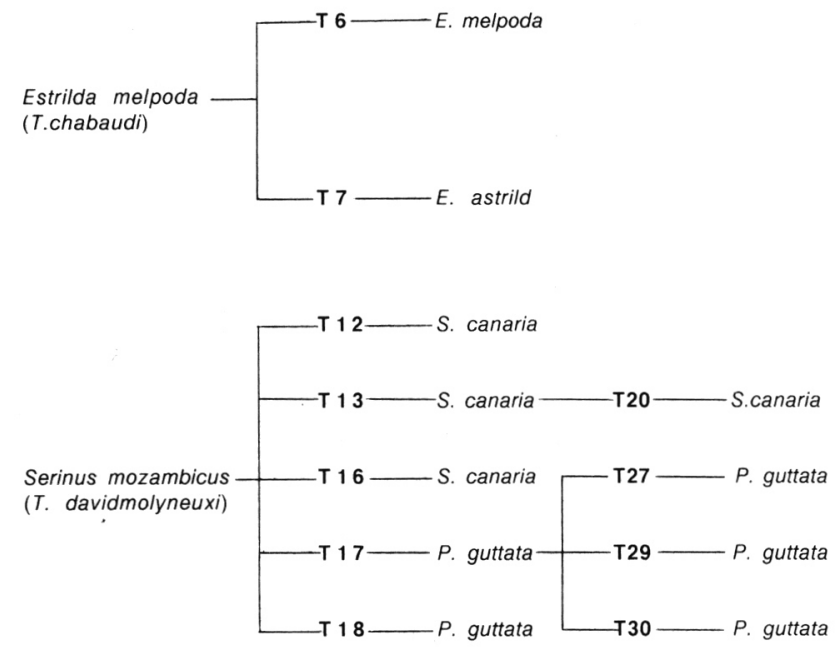

Fig. 2. - Espèces de Trypanosomes, hôtes naturels et hôtes expérimentaux infectés lors des transmissions avec Culicoides nubeculosus. $\mathrm{T}(\mathrm{N})=$ numéro de la transmission (cf. tableau I).

molyneuxi chez un Mandarin, en déposant des quantités croissantes de formes métacycliques (T22: $20000 \mathrm{mt}, \mathrm{T} 24$ : $25000 \mathrm{mt}$ et T26:50000 mt) sur les yeux de l'Oiseau. Les treize contrôles effectués pendant toute cette période ont été négatifs.

Afin d'éliminer un éventuel problème d'immunité ou de résistance naturelle de l'Oiseau utilisé, une injection par voie intra-péritonéale de 30000 formes métacycliques est réalisée huit jours (et quatre contrôles négatifs) après le dernier dépôt conjonctival. T. davidmolyneuxi est alors trouvé dans le sang, six jours plus tard, au premier examen sanguin suivant cette dernière injection.

Nous avions déjà testé la voie orale $(1988 b)$ avec $T$. chabaudi et observé après transmission, l'apparition d'un Trypanosome morphologiquement et physiologiquement différent de celui d'origine (cf. ci-dessus). Dans une nouvelle expérience de passage par cette voie d'un Serinus mozambicus à un Mandarin (T18) puis, de ce dernier à un autre Mandarin (T30), la morphologie et le comportement du parasite ont été normaux.

Enfin, chacune des autres voies, éprouvées seules, ont été efficaces au moins une fois T7 (IM), T27 (IP), T29 (SC).

\section{4 - Relation entre richesse de l'inoculum et taux de parasitémie}

L'analyse des expériences ne permet pas de déterminer la quantité minimale de formes métacycliques nécessaire à la détection de l'infection chez un Oiseau inoculé.

Il est possible de calculer approximativement le nombre théorique de Trypanosomes attendus en fonction de l'inoculum. En effet, si tous les parasites atteignent la circulation et, en estimant le volume total de sang de l'Oiseau à $1000 \mu \mathrm{l}$, l'inoculation, par exemple, de
25000 parasites (T29) entraînerait une parasitémie de $250 / 10 \mu \mathrm{l}$. Dans la réalité, la parasitémie est beaucoup plus faible, dépassant rarement $10 / 10 \mu \mathrm{l}$.

De nombreux paramètres entrent vraisemblablement en jeu et un même nombre de formes métacycliques peut induire une parasitémie chez un Oiseau alors qu'un autre reste négatif.

\section{DISCUSSION}

Le fait le plus inattendu au cours de notre étude est le faible rendement des infestations expérimentales. En effet, contrairement à ce qui est observé pour la plupart des hématozoaires à la suite d'une infestation expérimentale, les parasitémies sont presque toujours stables et relativement peu élevées.

Nous n'avons jamais observé de formes de multiplication dans le sang ou les capillaires des organes d'un Oiseau sacrifié. Les stades de division que nous avions décrits antérieurement, en 1988, provenaient d'Oiseaux trouvés morts dans leur cage et représentaient vraisemblablement des artefacts post mortem.

L'allure très stable et la faiblesse des parasitémies (dès la $4^{\mathrm{e}}$ heure après l'inoculation), dans les infections expérimentales chez l'Oiseau, semblent à notre avis, indiquer que les parasites inoculés se multiplient peu ou pas dans l'organisme. Les rares fluctuations d'amplitude, relativement faibles $(1$ à $25 / 10 \mu \mathrm{l})$ pourraient traduire des phénomènes de migration des parasites entre la circulation périphérique et les capillaires profonds. Quoi qu'il en soit, elles correspondent à des transformations biologiques qui entraînent une perte temporaire d'infectivité.

Le cas de l'expérience T6 mérite d'être examiné à part. Un passage avait été effectué (cf. plus haut) entre un Estrilda melpoda parasité par $T$. chabaudi et un autre Oiseau de la même espèce. Chez ce dernier, le parasite s'est fortement multiplié et la parasitémie a augmenté de façon exponentielle jusqu'à atteindre un taux de 1500 parasites par $10 \mu \mathrm{l}$ en trois mois. Cependant, cette multiplication anormale s'accompagnait de modifications morphologiques et d'une inaptitude à évoluer chez le Culicoïde, même lorsque, 22 mois plus tard, la parasitémie est redevenue stable et faible.

Nous avions alors interprété ce résultat comme la conséquence de l'absence chez le Culicoïde d'un facteur indispensable à la maturation des Trypanosomes qui, même s'ils peuvent se diviser chez l'Oiseau, ne sont plus capables d'infecter les Culicoïdes.

Compte tenu des résultats présentés ici, nous savons maintenant que les Trypanosomes transmis par Culicoïdes sont aussi infectants pour le vecteur et pour un nouvel Oiseau que ceux d'origine. $\mathrm{Si}$, comme nous le pensons, il n'y a pas, en règle générale, de multiplication dans le sang de l'Oiseau, l'évolution de $T$. chabaudi dans l'expérience T6 était aberrante. 


\section{RÉFÉRENCES}

Baker J. R. : Biology of the Trypanosomes of Birds. In: Lumsden W. H. R. and Evans D. A., Biology of kinetoplastida, vol. 1. Academic Press Inc., London, 1976, 131-174.

Bennett G. F. : On the specificity and transmission of some avian Trypanosomes. Can. J. Zool., 1961, 39, 17-33.

Boorman J. : The maintenance of laboratory colonies of Culicoides variipennis (Coq.), C. nubeculosus (Mg.) and C. riethi Kieff. (Diptera, Ceratopogonidae). Bull. Ent. Res., 1974, 64, 371-377.

Chandenier J., Landau I., Baccam D. : Sur les Trypanosomes d'oiseaux Estrildidae. I. Étude morphologique et systématique. Ann. Parasitol. Hum. Comp., 1988 a, 63, 184-192.

Chandenier J., Landau I., Baccam D. : Sur les Trypanosomes d'oiseaux Estrildidae. II. Études biologiques. Ann. Parasitol. Hum. Comp., 1988 b, 63, 243-252.

Miltgen F., Landau I. : Culicoides nubeculosus, vecteur expérimental d'un nouveau Trypanosome de Psittaciforme : Trypanosoma bakeri n. sp. Ann. Parasitol. Hum. Comp., 1982, 57, 423-428.

Miltgen F., Landau I. : Trypanosoma johnbakeri, nomen novum pour Trypanosoma bakeri Miltgen et Landau, 1982. Ann. Parasitol. Hum. Comp., 1988, 63, 196. 